Z Gerontol Geriat 2014 · 47:4-5

DOI 10.1007/s00391-013-0593-6

Online publiziert: 5. Januar 2014

(c) Springer-Verlag Berlin Heidelberg 2014

Manfred Gogol

Klinik für Geriatrie, Krankenhaus Lindenbrunn, Coppenbrügge

\title{
Geriatrie in einem sich verändernden Versorgungssystem
}

lin, Bremen und Hamburg mit 17 Betten dar. Auch im Bereich nach $\$ 111$ SGB V sank die Auslastung kontinuierlich von 86,9 auf $78,7 \%$ [1].

In ihrem Beitrag „10 Jahre geriatrische frührehabilitative Komplexbehandlung im DRG-System“ bewerten G. Kolb et al. die Einführung und Etablierung der geriatrischen frührehabilitativen Komplexbehandlung in das deutsche DRG-System aus Sicht der Leistungserbringer, des Medizinischen Dienstes und der Kostenträger und zeigen Stärken und Schwächen auf. Insbesondere die Definition spezifischer Qualitäts- und Abgrenzungskriterien hinsichtlich der Operationalisierung von Krankenhausbehandlungsbedürftigkeit und der Übergang vom Krankenhaus- in den Rehabilitationsbereich ist bisher nicht zufriedenstellend geregelt.

Konzeptionell entwickeln D. van den Heuvel et al. in ihrer Arbeit „Geriatrische Versorgungsstrukturen in Deutschland“ das Konzept eines integrierten geriatrischen Versorgungsverbundes, der es ermöglicht, die verschiedenen Sektoren der Versorgung älterer Patienten patientenzentriert zusammenzufassen und die Schwächen des sowohl streng sektoralen wie der heterogenen Platzierung der Geriatrie in den einzelnen Bundesländern zu berücksichtigen.

Trotzdem muss es ein Anliegen der Geriatrie und seiner Vertreter auch in der heutigen Versorgungssituation sein, die fachliche Qualität der Leistungserbringung an die erste Stelle zu setzen. Es verwundert sehr, wenn in einem Bundesland von einer geriatrischen Fachabteilung ein
Oberarzt in einer Stellenanzeige gesucht wird, der explizit die Zusatzbezeichnung nicht aufweisen muss, aber zugleich die Therapiekonferenzen leiten soll [2].

Auch ist in den letzten zwei, drei Jahren zu beobachten, dass Akutkrankenhäuser vermehrt die Frührehabilitationsprozedur OPS 8.550.x abrechnen oder sogar geriatrische Fachabteilungen einrichten. Dies scheint aber nicht immer von der Intention getragen zu sein, die medizinische Versorgung älterer Patienten zu verbessern, sondern ist möglicherweise einer primär wirtschaftlichen Analyse der eigenen Kliniksituation geschuldet. In Niedersachsen ist jetzt einem Chefarzt kurzfristig gekündigt worden, der mit dem Ziel angetreten war, eine neue Krankenhausabteilung aufzubauen [3]. Die Kündigung erfolgte nachdem ein privater Krankenhausträger den anderen gekauft hatte. Analysiert man die lokale Situation, ist zu erkennen, dass dann mehrere geriatrische Fachabteilungen auf engem Raum in einem Flächenland mit relativ geringer Einwohnerzahl wirtschaftlich nicht vom gleichen Träger erfolgreich zu betreiben sein werden - folgerichtig, so könnte man konstatieren. Zugleich ist es aber ein Indiz dafür, dass die Implementierung von Altersmedizin in deutschen Krankenhäusern nicht immer primär das Ziel der besseren Patientenversorgung verfolgen muss. Hier kann sich zukünftig ein erhebliches Problemfeld entwickeln.

Vielerorts ist weiterhin die Anbindung der geriatrischen Rehabilitation an das bestehende System der Krankenhausver- 
sorgung problematisch. Hierzu haben C. Becker und D. van den Heuvel in dieser Zeitschrift eine provokante Stellungnahme verfasst [8]. Auch hier steht die deutsche Geriatrie vor einer Diskussion, der man nicht mehr auszuweichen kann. Es ist sicher häufig möglich und angemessen, geriatrische Patienten im Krankenhausbereich fallabschließend zu behandeln. Trotzdem muss empirisch erwartet werden, dass auch aus einem solchen Setting ein Transfer in eine weitere rehabilitative Behandlung erfolgt. Wenn aber eine geriatrische Fachabteilung in Niedersachsen, einem Bundesland mit primär parallel im Krankenhaus- und Rehabilitationsbereich aufgestellter Geriatrie, damit wirbt, dass nur „in Ausnahmefällen ... eine Anschlussrehabilitation möglich“ sein kann, dann erscheinen die eigenen Vorstellungen von Behandlungsqualität und -erfolg omnipotent und nicht patientenzentriert zu sein [4].

Diese Zeilen lassen erkennen, dass längst die Ökonomie - zumindest in manchen Bereichen - Taktgeber der Patientenversorgung geworden ist. Im zweiten Teil dieses Schwerpunktes behandelt deshalb D. Strech in seinem Beitrag „Der Abbau von Überversorgung als Teil der ärztlichen Berufsethik" das Thema der Überversorgung im Gesundheitswesen. Anlass ist die Initiative des American Board of Internal Medicine (ABIM) zur Choosing Wisely-Initiative [5]. Diese hat zum Ziel, dass jede Fachgesellschaft eine Top-5-Liste erstellt, um Maßnahmen $\mathrm{zu}$ reduzieren, die unnötig sind, dem $\mathrm{Pa}$ tienten schaden können und Kosten verursachen.

Die Vorschläge der American Geriatric Society [6] und der American Medical Director Association [7] werden von M. Gogol in seinem Beitrag „Choosing Wisely“ vorgestellt. Die grundsätzliche Idee hinter der ABIM-Initiative ist zu begrüßen, doch ist zu prüfen, ob eine solche Initiative auch in der deutschen Versorgungskultur realisiert werden kann.

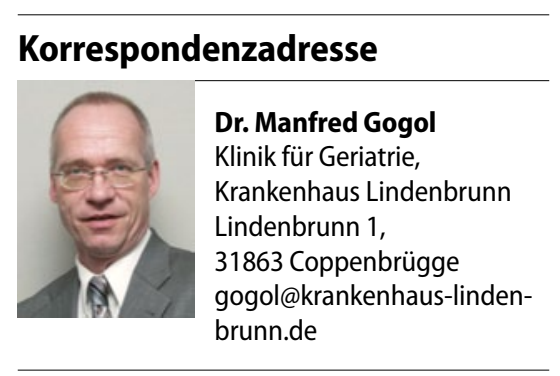

Interessenkonflikt. M. Gogol gibt an, dass kein Interessenkonflikt besteht.

\section{Literatur}

1. Böhm K (2013) Gesundheit und soziale Sicherung. In: Statistisches Bundesamt (Hrsg.) Datenreport 2013. Ein Sozialbericht für die Bundesrepublik Deutschland. Bonn:217-248

2. Ärztestellen(2013) http://www.aerzteblatt.de/ aerztestellen/anzeige?sort=datum+desc\&stellenVolltext=geriatrie\&id=420889. Zugegriffen: 29.November 2013

3. Helios-Kliniken (2013) Chefarzt für Geriatrie startet. http://www.helios-kliniken.de/pdf/klinik/ northeim/aktuelles/news-detail/archiv/2013/august/artikel/chefarzt-fuer-geriatrie-startet.html. Zugegriffen: 29.11.2013

4. Helios-Kliniken (2013) http://www.helios-kliniken. de/klinik/bad-gandersheim/fachabteilungen/innere-medizin/klinische-geriatrie/alterstraumatologie.html. Zugegriffen: 29.11.2013

5. ABIM Foundation (2013) http://www.abimfoundation.org/Initiatives/Choosing-Wisely.aspx. Zugegriffen: 29.11.2013

6. AGS Choosing Wisely Workgroup (2013) American Geriatrics Society identifies five things that healthcare providers and patients should question. J Am Geriatr Soc 61:622-631

7. Vance J (2013) AMDA - Choosing Wisely. J Am Med Dir Assoc 14:639-641

8. Becker C, van den Heuvel D (2013) Geriatrische Rehabilitation. Ein Modell ohne Zukunft? Z Gerontol Geriat 46:489-490 